\title{
Meeting synopsis: advances in skeletal muscle biology in health and disease (Gainesville, Florida, February 22nd to 24th 2012) - day 1: “cell signaling mechanisms mediating muscle atrophy and hypertrophy" and "muscle force, calcium handling, and stress response"
}

\author{
Andrew R. Judge ${ }^{1,2 *}$, Scott K. Powers' ${ }^{2}$, Leonardo F. Ferreira ${ }^{2}$ and Marcas M. Bamman ${ }^{3}$ \\ Department of Physical Therapy, University of Florida, Gainesville, FL, USA \\ 2 Department of Applied Physiology and Kinesiology, University of Florida, Gainesville, FL, USA \\ 3 UAB Center for Exercise Medicine, University of Alabama at Birmingham, Birmingham, AL, USA \\ ${ }^{*}$ Correspondence: arjudge@phhp.ufl.edu
}

The "Advances in Skeletal Muscle Biology in Health and Disease" conference was held in Gainesville, Florida February 22nd to 24th 2012. The primary purpose of this meeting was to facilitate advances in adult skeletal muscle biology and physiology. There were 210 registered participants from 64 different institutions and 4 pharmaceutical companies, including participants from 10 different countries. Approximately $40 \%$ of the attendees and one-third of the symposium speakers were women. The meeting began with a grant writing workshop that included an overview of training grants, R01 grant structure, and grants scoring and interpreting reviewer comments given by Drs. Tom Clanton, Denis Guttridge, Marcas Bamman, and Sue Bodine. This workshop was followed by a poster session that included 65 outstanding posters presented by faculty, postdoctoral fellows, and graduate students. The symposium consisted of 28 total speakers across four main sessions. In this two part synopsis we outline some of the main findings, current paradigms, and future directions presented in each of these sessions. In Part 1, we outline Day 1 of the symposium which consisted of sessions on "Cell signaling mechanisms mediating muscle atrophy and hypertrophy" and "Muscle force, calcium handling, and stress response." In Part 2, we outline Day 2 of the symposium which consisted of sessions on "Muscle diseases and regeneration" and "Clinical/translational research."

\section{CELL SIGNALING MECHANISMS MEDIATING MUSCLE ATROPHY AND HYPERTROPHY}

Considering the powerful impact of skeletal muscle mass on health status, and the deleterious consequences of the muscle atrophy that accompanies numerous chronic disease states, it should come as no surprise that a major focus of this meeting was on fundamental aspects of muscle mass regulation. At its roots, skeletal muscle mass is determined by the constant interplay between mechanisms that drive protein synthesis vs. those that promote protein degradation. The first and largest session of this meeting thus included presentations by 11 scientists prominent in the field of muscle mass regulation who have taken advantage of animal models to reveal key cellular and molecular mechanisms that mediate muscle atrophy and hypertrophy. These speakers included Drs. Sue Bodine, Susan Kandarian, Andrew Judge, Charlotte Peterson, Yi-Ping Li, Teresa Zimmers, Jim Carson, Mike Reid, Zhen Yan, Scott Powers, and Esther Dupont-Versteegden.

Like all other sessions, these speakers described the current state of knowledge in specific niches while highlighting their findings and contributions to the field. A few of these presentations are highlighted here. For example, Dr. Bodine opened the symposium by presenting her work on the role of MuRF1 in skeletal muscle atrophy. MuRF1 and MAFbx were identified in 2001 as atrophy-associated genes. In the past 10 years, both genes have been shown to be upregulated under a variety of atrophy-inducing conditions. Data were presented showing that deletion of MuRF1, but not MAFbx, results in muscle sparing under a variety of atrophy-inducing conditions. Further, inhibition of MAFbx was not required to get muscle sparing in the MuRF1 KO mice. Contrary to what has been predicted, deletion of MuRF1 resulted in an increase in $20 \mathrm{~S}$ and $26 \mathrm{~S}$ proteasome activity under certain atrophy-inducing conditions. These new data suggest that the in vivo targets of MuRF1 may not be restricted to the thick filament proteins, and suggest possible roles in transcription and protein synthesis.

Dr. Kandarian presented data on NF-kappaB signaling in skeletal muscle atrophy. The transcription factors $\mathrm{p} 50$ and $\mathrm{Bcl}-3$ are required for hind limb unloading induced muscle atrophy and unloading induced activation of NF-kappaB reporter activity. That is, NF-kappaB activity is abolished and fiber atrophy completely prevented in unloaded muscle in mice lacking p50 or Bcl-3. To understand the cellular regulation of disuse atrophy Dr. Kandarian's laboratory identified downstream target genes of p50 and $\mathrm{Bcl}-3$ transcription factors using global gene expression microarrays in wild type and knockout mice for these two factors. All genes identified as p50 target genes were a subset of the genes identified as Bcl-3 target genes, suggesting they may act together in regulating gene expression during muscle atrophy. In order to identify genes that have increased $\mathrm{Bcl}-3$ binding on a genome-wide basis and thus are $\mathrm{Bcl}-3$ direct downstream target genes during atrophy, Dr. Kandarian used ChIP-sequencing on chromatin isolated from control and 5 day unloaded muscle. Compared to control, there was increased Bcl-3 binding in unloaded muscle in families of genes that regulate proteolysis, while p50 binding was often found in the same gene locations but to a similar extent in control and unloaded muscle. Dr. Kandarian summarized that, as the transactivator in $\mathrm{p} 50-\mathrm{Bcl}-3$ complexes, Bcl-3 
binding to target genes found in atrophying muscle further supports its role as an essential regulator of the genes causing muscle atrophy due to disuse.

Dr. Peterson discussed and presented data to show that in satellite cell-depleted mouse muscle, robust fiber hypertrophy, and re-growth following atrophy occur independent of myonuclear accretion. These novel findings challenge the popular view that myofiber hypertrophy is facilitated by, or dependent upon, satellite cell-mediated myonuclear addition. Further, the results indicate no other cell population is replacing satellite cells in this myonuclear addition role. However, muscle regeneration is impaired in satellite cell-depleted mouse muscle, showing that muscle fiber regeneration and hypertrophy have distinct satellite cell requirements. Dr. Peterson concluded by posing some open questions for discussion, including "Can the mechanisms that enable mouse muscle to grow in the absence of satellite cells be activated in human muscle as therapeutic targets for sarcopenia, cachexia, or muscle wasting?" "Are there species-specific differences in the flexibility of the myonuclear domain?" and, "Are there technical limitations to quantification of satellite cell fusion/myonuclear dynamics in adult muscle?"

Dr. Li presented his findings showing that Lewis lung carcinoma cells release soluble factors which induce ubiquitin ligase atrogin1/MAFbx upregulation and muscle catabolism. Carcinoma-induced atrogin1/MAFbx upregulation is mediated by $\mathrm{p} 38 \beta$ MAPK activation of transcription factor $\mathrm{C} / \mathrm{EBP} \beta$ binding to the atrogin1/MAFbx promoter via selective phosphorylation, and is independent of FoxO1/3. Further, muscle wasting induced by tumor xenograft is blocked in $\mathrm{C} / \mathrm{EBP} \beta$ null mice or mice administered an inhibitor of $\mathrm{p} 38 \alpha / \beta$ MAPK. Dr. Li concluded that the $\mathrm{p} 38 \beta \mathrm{MAPK}-\mathrm{C} / \mathrm{EBP} \beta$ signaling pathway appears to be a therapeutic target for cancer cachexia.

Dr. Zimmers also presented data on cancer cachexia and began by emphasizing that although IL- 6 and related ligands have been implicated in muscle wasting of cancer, the downstream signaling pathways responsible are unknown. Dr. Zimmers subsequently presented data indicating that STAT3 is causally involved in muscle wasting in vitro and in vivo. Inhibition of STAT3 by various mechanisms reduced muscle wasting in conditions of high IL-6 or cancer.

Dr. Yan presented evidence that in vivo administration of the endogenous $\mathrm{NO}$ donor, S-Nitroso-L-glutathione, induces Extracellular Superoxide Dismutase (EcSOD) expression and provides protection against dexamethasone-induced muscle atrophy. Both somatic gene transfer of EcSOD and transgenic overexpression of EcSOD are sufficient to render protection against catabolic muscle wasting. Dr. Yan also presented evidence that muscle-derived EcSOD expression in transgenic mice provides profound protection against multiple organ dysfunction syndrome (MODS) induced by endotoxemia.

Dr. Dupont-Versteegden presented her recent data showing failed muscle regrowth in the muscles of old vs. young rats. Specifically, the soleus muscle of 6- and 32 -month-old rats atrophied in response to 2 weeks of hind limb suspension but only muscles from the 6 month old returned to control levels following 2 weeks of reloading. mRNA abundance of protein degradation marker MAFbx and autophagy markers Atg5 and Atg7 increased with hind limb suspension and were restored to control with reloading in soleus muscle of young and old rats. Similarly, phosphorylated GSK3beta was decreased with atrophy and returned to control upon reloading in both ages. In contrast, no changes in phosphorylated p70s6 kinase were observed at either age. Dr. Dupont-Versteegden concluded that changes in anabolic and catabolic pathways associated with alterations in muscle size are similar in soleus muscles of young and old rats, suggesting that factors external to muscle may play a role in the attenuated muscle re-growth response in the aged.

\section{MUSCLE FORCE, CALCIUM HANDLING, AND STRESS RESPONSE}

Beyond its mass, the force generating capacity of skeletal muscle is highly dependent on, among other factors, the integrity of the excitation-contraction coupling system and mitochondrial function. Recent advances of molecular biology and imaging technologies have markedly expanded our understanding of these critical modulators of muscle force. The presentations of the second session therefore highlighted recent contributions to this field by Drs. Håkan
Westerblad, Leonardo Ferreira, and Tom Clanton. Dr. Westerblad presented work from his laboratory showing that $\mathrm{Ca}^{2+}$ plays an important role in the interaction between mitochondria and general skeletal muscle cell function. The concentration and location of $\mathrm{Ca}^{2+}$ is critical for the physiological effects. Indeed, findings from the Westerblad laboratory show that a limited $\mathrm{Ca}^{2+}$ leak from the sarcoplasmic reticulum (SR) is accompanied by increased mitochondrial biogenesis and increased fatigue resistance, whereas a larger SR $\mathrm{Ca}^{2+}$ leak results in muscle weakness. On the other hand, muscle fibers of mice with a specific mitochondrial myopathy accumulate $\mathrm{Ca}^{2+}$ in the mitochondria, which is accompanied by decreased SR $\mathrm{Ca}^{2+}$ content and muscle weakness. These complex $\mathrm{Ca}^{2+}$-dependent interactions were discussed.

Dr. Ferreira presented his work on sphingolipids, oxidants, and skeletal muscle contractile dysfunction in heart failure. Sphingomyelinase (SMase) catalyzes the conversion of sphingomyelin to ceramide and phosphorylcholine. Ceramide acts as a second messenger that activates various signaling pathways and increases oxidants in the cell. SMase activity is heightened in patients with congestive heart failure (CHF) and is inversely related to skeletal muscle force. Dr. Ferreira presented preliminary data suggesting that SMase activity and ceramide content are heightened in the skeletal muscle of CHF rats. Exogenous exposure of intact skeletal muscle to SMase in vitro decreases force and this effect is prevented by antioxidants. The decrease in force elicited by SMase was also evident in skinned single fibers, which showed lower maximal force and calcium sensitivity of the contractile apparatus. Dr. Ferreira concluded that SMase and ceramide promote oxidantmediated muscle weakness. Thus, increased SMase activity and ceramide content may contribute to contractile dysfunction in CHF.

The first day ended with an informal dinner, attended by 176 of the registrants, where friendly scientific discussions on adult muscle plasticity continued throughout the evening.

\section{ACKNOWLEDGMENTS}

The University of Florida organizers (ARJ, SKP, LFF) gratefully acknowledge the financial support of: National Institutes of Health, 
National Center for Medical Rehabilitation Research grant T32 DO043730; University of Florida: Department of Physical Therapy, Center for Exercise Science and Department of Applied Physiology and Kinesiology, College of Public Health and Health Professions, and College of Health and Human Performance; Rigel Pharmaceuticals; Aurora Scientific, Inc.; and World Precision Instruments. This meeting was endorsed by the American Physiological Society.

Received: 07 May 2012; accepted: 22 May 2012; published online: 11 June 2012.

Citation: Judge AR, Powers SK, Ferreira LF and Bamman MM (2012) Meeting synopsis: advances in skeletal muscle biology in health and disease (Gainesville, Florida, February

22nd to 24th 2012) - day 1: "cell signaling mechanisms mediating muscle atrophy and hypertrophy" and "muscle force, calcium handling, and stress response." Front. Physio. 3:200. doi: 10.3389/fphys.2012.00200

This article was submitted to Frontiers in Striated Muscle Physiology, a specialty of Frontiers in Physiology.

Copyright $\odot 2012$ Judge, Powers, Ferreira and Bamman. This is an open-access article distributed under the terms of the Creative Commons Attribution Non Commercial License, which permits non-commercial use, distribution, and reproduction in other forums, provided the original authors and source are credited. 